\title{
Impact of five annual rounds of mass drug administration with ivermectin on onchocerciasis in Sierra Leone
}

\author{
Joseph B. Koroma', Santigie Sesay², Abdul Conteh², Benjamin Koudou³, Jusufu Paye ${ }^{4}$, Mohamed Bah",
} Mustapha Sonnie $^{4}$, Mary H. Hodges ${ }^{4}$, Yaobi Zhang ${ }^{5^{*}}$ (D) and Moses J. Bockarie ${ }^{6}$

\begin{abstract}
Background: Onchocerciasis is endemic in 12 of the 14 health districts of Sierra Leone. Good treatment coverage of community-directed treatment with ivermectin was achieved between 2005 and 2009 after the 11-year civil conflict. Sentinel site surveys were conducted in 2010 to evaluate the impact of five annual rounds of ivermectin distribution.
\end{abstract}

Methods: In total, 39 sentinel villages from hyper- and meso-endemic areas across the 12 endemic districts were surveyed using skin snips in 2010. Results were analyzed and compared with the baseline data from the same 39 villages.

Results: The average microfilaridermia (MF) prevalence across 39 sentinel villages was $53.10 \%$ at baseline. The MF prevalence was higher in older age groups, with the lowest in the age group of 1-9 years (11.00\%) and the highest in the age group of 40-49 years (82.31\%). Overall mean MF density among the positives was 28.87 microfilariae $(\mathrm{mf}) / \mathrm{snip}$, increasing with age with the lowest in the age group of 1-9 years and the highest in the age group of 40-49 years. Males had higher MF prevalence and density than females. In 2010 after five rounds of mass drug administration, the overall MF prevalence decreased by $60.26 \%$ from $53.10 \%$ to $21.10 \%$; the overall mean MF density among the positives decreased by $71.29 \%$ from $28.87 \mathrm{mf} / \mathrm{snip}$ to $8.29 \mathrm{mf} / \mathrm{snip}$; and the overall mean MF density among all persons examined decreased by $88.58 \%$ from $15.33 \mathrm{mf} / \mathrm{snip}$ to $1.75 \mathrm{mf} / \mathrm{snip}$. Ten of 12 endemic districts had $>50 \%$ reduction in MF prevalence. Eleven of 12 districts had $\geq 50 \%$ reduction in mean MF density among the positives.

Conclusions: A significant reduction of onchocerciasis MF prevalence and mean density was recorded in all 12 districts of Sierra Leone after five annual MDAs with effective treatment coverage. The results suggested that the onchocerciasis elimination programme in Sierra Leone was on course to reach the objective of eliminating onchocerciasis in the country by the year 2025. Annual MDA with ivermectin should continue in all 12 districts and further evaluations are needed across the country to assist the NTDP with programme decision making.

Keywords: Onchocerciasis, Onchocerca volvulus, Mass drug administration, Community-directed treatment with ivermectin, Community-directed drug distributor, Skin snip, Onchocerciasis-endemic, Post-conflict, Disease elimination, Rapid diagnostic test

\footnotetext{
* Correspondence: yzhang@hki.org

${ }^{5}$ Helen Keller International, Regional Office for Africa, Dakar, Senegal

Full list of author information is available at the end of the article
} International License (http://creativecommons.org/licenses/by/4.0/), which permits unrestricted use, distribution, and reproduction in any medium, provided you give appropriate credit to the original author(s) and the source, provide a link to the Creative Commons license, and indicate if changes were made. The Creative Commons Public Domain Dedication waiver (http://creativecommons.org/publicdomain/zero/1.0/) applies to the data made available in this article, unless otherwise stated. 


\section{Multilingual abstracts}

Please see Additional file 1 for translations of the abstract into the five official working languages of the United Nations.

\section{Background}

Onchocerciasis, also known as river blindness or Robles' disease, is a parasitic disease caused by infection with the parasite Onchocerca volvulus. The disease is transmitted to humans by the black fly (Simulium spp.) and its pathology is linked to the death of the microfilariae in the skin and eyes [1-3]. Humans are known as the main reservoirs for $O$. volvulus [4]. Some animals such as elands and buffalos are possible reservoir hosts, which makes control of the disease in areas where these animals co-exist more difficult [5]. Currently, there are an estimated 187 million people at risk of onchocerciasis among which 37 million are infected with O. volvulus. Among those infected, an estimated 4 million people live with skin manifestations of the disease and 2 million are estimated to be either visually impaired or blind [1-4]. Onchocerciasis is the world's second leading infectious cause of blindness after trachoma. Blindness from onchocerciasis occurs early in life (20-30 years old) and therefore creates socio-economic problems for those affected, their families and their communities [1-4]. About $99 \%$ of reported cases of the disease are in 31 endemic countries in sub-Saharan Africa [1, 3, 6-8].

Onchocerciasis control in Africa started with the launching of the Onchocerciasis Control Programme in West Africa (OCP) in seven countries (Benin, Burkina Faso, Cote d'Ivoire, Ghana, Mali, Niger and Togo) in 1974 focusing on vector control and then continued with the African Programme for Onchocerciasis Control (APOC) in 1995 focusing on community-directed treatment with ivermectin (CDTI) in meso- (microfilaridermia [MF] prevalence 40\%-59.9\%) and hyper- (MF prevalence $\geq 60 \%$ ) endemic sites [9]. Since 2009 there has been a paradigm shift from control of onchocerciasis as a public health problem (reduction of O. volvulus MF prevalence to an acceptable low level where transmission may continue) to elimination of the disease by stopping local transmission. This shift was motivated by studies in Senegal and Mali which demonstrated that through treatment with ivermectin it was possible to eliminate the disease [10]. It is currently believed that below 5\% O. volvulus MF prevalence the disease prevalence would continue to drop even in the absence of treatment and transmission would stop eventually $[4,10]$. The recommended programme coverage (i.e. proportion of people ingesting ivermectin among people targeted in the endemic districts that are eligible for treatment, i.e. $\geq 5$ years) during treatment with ivermectin is $\geq 80 \%$ in all endemic areas [3, 4, 10-13].
The endemicity of onchocerciasis was demonstrated in Sierra Leone in 1926 when Blacklock first described its transmission through the black fly ( $S$. damnosum) in the Kono district [14]. Onchocerciasis control efforts in the country started as early as in 1957 with insecticide treatments along the Tonkolili River that was found to be the most severely affected [15]. It was documented that onchocerciasis was the second most common cause of blindness after cataracts in Sierra Leone and in the late 1980s the former OCP extended its activities to four other countries - Guinea, Guinea-Bissau, Senegal and Sierra Leone [16-19]. Considerable work since the 1950s demonstrated high onchocerciasis prevalence in Sierra Leone along the main rivers and existence of black flies in the entire country except in areas around the capital of Freetown and the southern coastal plain of the Bonthe district [20]. During 1988-2004, surveys from 177 sites across all 14 districts, selected based on proximity to rivers and surveyed using the skin snip method, showed that the unadjusted MF prevalence varied from $0 \%$ to $78.3 \%$ (see Fig. 1) [20]. With technical assistance from APOC, villages around survey sites were classified to be hypo-, meso- or hyper-endemic according to the adjusted MF prevalence. The historical data and timelines of onchocerciasis control activities in Sierra Leone is summarized in Table 1.

The National Onchocerciasis Control Programme (NOCP) in Sierra Leone was established in 1989 under the OCP. However, the civil conflict between 1991 and 2002 negatively impacted on onchocerciasis control activities, and from 1997 to 2002 only limited onchocerciasis control activities were conducted in areas with high prevalence and with security; therefore, treatment coverage before 2002 was deemed not reliable. With financial and technical support from APOC, the NOCP restarted interventions in 2003 through the Special Intervention Zones (SIZ) programme (2003-2007). CDTI was implemented in meso- and hyper-endemic areas in the 12 endemic districts [17-19, 21]. However, epidemiological coverage (i.e. proportion of people ingesting ivermectin among the total population in the targeted endemic districts) was reported to be $36 \%$ and $28 \%$ in 2003 and 2004 respectively, and geographic coverage (i.e. proportion of endemic communities and districts targeted among all the endemic communities and districts needing treatment) could not be determined for the two years [20]. Efforts to control onchocerciasis improved in 2005, in part due to changes effected within the programme management by the Ministry of Health and Sanitation (MOHS) to improve onchocerciasis control efforts in the country [20]. Treatment coverage has significantly improved since 2005.

In 2007, the NOCP was expanded to become the national integrated Neglected Tropical Disease Programme 


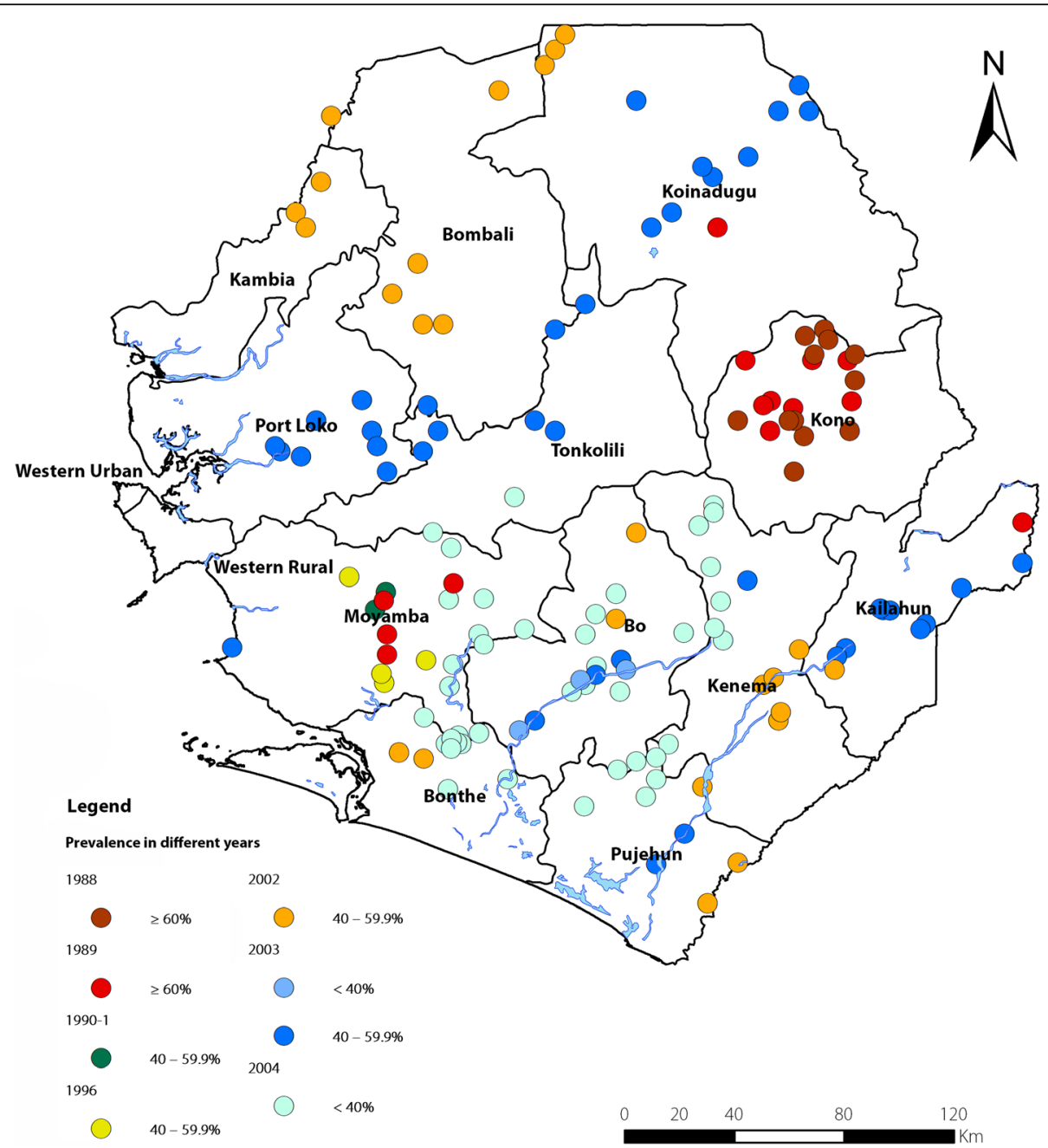

Fig. 1 Distribution map of unadjusted point prevalence of onchocerciasis from baseline surveys in Sierra Leone. Data were collected during 1988 and 2004. Prevalence results from different years are plotted separately

(NTDP) to include onchocerciasis, lymphatic filariasis (LF), schistosomiasis and soil-transmitted helminths. LF was endemic in all 14 districts and co-endemic with onchocerciasis in 12 districts. Therefore, co-administering albendazole and ivermectin in all LF endemic districts effectively treated all hyper-, meso- and hypo-endemic villages for onchocerciasis.

The current World Health Organisation (WHO) guidelines on onchocerciasis elimination recommend impact assessment following 3-5 annual rounds of effective ivermectin distribution [22]. In line with these recommendations, a national sentinel site assessment was conducted in 2010-2011 using the skin snip method. The purpose of this paper is to determine the impact of five annual rounds of ivermectin distribution on the transmission of onchocerciasis. We compared the data from sentinel sites across the 12 onchocerciasis-endemic districts at baseline and in 2010-2011 and discussed the way forward in reaching onchocerciasis elimination in Sierra Leone.

\section{Methods}

Mass drug administration

Annual CDTI started in 2003. However, effective treatment coverage was not achieved until 2005 due to uncertainty around population data post-war and the internal migration of displaced people. Integrated, annual onchocerciasis/LF mass drug administration (MDA) with ivermectin and albendazole was piloted in 2007 in six districts located in border areas with neighbouring Guinea and Liberia, while CDTI continued in the other six districts. Integrated onchocerciasis/LF MDA was scaled up to cover all 12 coendemic districts in 2008. Community-directed drug distributors (CDDs) used dose poles to distribute ivermectin and later ivermectin plus albendazole to the eligible population aged five years and older. The CDDs were literate members of communities that were selected by their communities. District health workers conducted training of CDDs for MDA and provided supervision during MDA. 
Table 1 Historical data and time lines of onchocerciasis control activities in Sierra Leone

\begin{tabular}{|c|c|c|}
\hline Year & Event & Comments \\
\hline 1926 & $\begin{array}{l}\text { The endemicity of onchocerciasis was demonstrated in Sierra } \\
\text { Leone when Blacklock first described its transmission through } \\
\text { the black fly, S. damnosum in the Kono district }\end{array}$ & \\
\hline 1957 & $\begin{array}{l}\text { Start of onchocerciasis control efforts with insecticide treatment } \\
\text { along the Tonkolili River that was found to be the most } \\
\text { severely affected }\end{array}$ & \\
\hline 1974 & $\begin{array}{l}\text { Launch of the Onchocerciasis Control Programme in West } \\
\text { Africa (OCP) in } 7 \text { countries (Benin, Burkina Faso, Cote d'Ivoire, } \\
\text { Ghana, Mali, Niger and Togo) }\end{array}$ & $\begin{array}{l}\text { Focus was on vector control but chemotherapy was added in } \\
\text { the late } 1980 \text { s and early 2000s }\end{array}$ \\
\hline 1988/9 & $\begin{array}{l}\text { OCP was extended to four other countries - Guinea, Guinea- } \\
\text { Bissau, Senegal and Sierra Leone }\end{array}$ & \\
\hline 1989 & $\begin{array}{l}\text { The National Onchocerciasis Control Program (NOCP) was } \\
\text { established in Sierra Leone under the OCP }\end{array}$ & \\
\hline 1995 & $\begin{array}{l}\text { Launch of the African Programme for Onchocerciasis Control } \\
\text { (APOC) }\end{array}$ & $\begin{array}{l}\text { Initiated the community-directed treatment with ivermectin } \\
\text { (CDTI) strategy }\end{array}$ \\
\hline $1991-2002$ & $\begin{array}{l}\text { Civil conflict in Sierra Leone resulted in limited onchocerciasis } \\
\text { activities }\end{array}$ & Treatment coverage before 2002 are considered unreliable \\
\hline 2003 & $\begin{array}{l}\text { Onchocerciasis control activities was restarted as part of the } \\
\text { Special Intervention Zones (SIZ) programme (2003-2007) that } \\
\text { was managed by APOC }\end{array}$ & $\begin{array}{l}\text { CDTI was implemented in meso- and hyper-endemic areas of } \\
\text { the } 12 \text { endemic districts with very poor epidemiological and } \\
\text { geographic coverage in 2003-2004 }\end{array}$ \\
\hline 1998-2005 & $\begin{array}{l}\text { Epidemiological mapping was conducted with support from } \\
\text { APOC, showing high onchocerciasis prevalence along the main } \\
\text { rivers and existence of black flies in the entire country except in } \\
\text { areas around the capital Freetown and the southern coastal } \\
\text { plain of the Bonthe district }\end{array}$ & $\begin{array}{l}\text { A total of } 177 \text { sites were surveyed using skin snip method } \\
\text { across } 14 \text { districts in the country. }\end{array}$ \\
\hline 2005 & $\begin{array}{l}\text { Management changes made within the NOCP by the Ministry } \\
\text { of Health and Sanitation (MOHS) to improve onchocerciasis } \\
\text { control efforts in the country }\end{array}$ & Better treatment coverage reported since then \\
\hline 2007 & $\begin{array}{l}\text { The NOCP expanded to become the national integrated } \\
\text { Neglected Tropical Disease Programme (NTDP) to include } \\
\text { onchocerciasis, lymphatic filariasis (LF), schistosomiasis and soil- } \\
\text { transmitted helminths. Trachoma was demonstrated to be non- } \\
\text { endemic in Sierra Leone. }\end{array}$ & $\begin{array}{l}\text { Onchocerciasis and LF activities integrated since then in all } 12 \\
\text { co-endemic districts by co-administering albendazole and iver- } \\
\text { mectin treating all hyper-, meso- and hypo-endemic villages } \\
\text { for onchocerciasis. }\end{array}$ \\
\hline 2009 & $\begin{array}{l}\text { Paradigm shift from control of onchocerciasis as a public health } \\
\text { problem (reduction of } O \text {. volvulus microfilaridermia prevalence } \\
\text { to an acceptable low level although transmission will continue) } \\
\text { to eliminating the disease by stopping local transmission }\end{array}$ & $\begin{array}{l}\text { This was after studies in Senegal and Mali showed that } \\
\text { through treatment with ivermectin it is possible to eliminate } \\
\text { the disease }\end{array}$ \\
\hline
\end{tabular}

NTDP staff and members of the district health management teams (DHMTs) also supported training and supervision of the MDA.

Distribution of medicines was recorded using community registers. The register was designed to capture all members of each community targeted for treatment. Before each MDA, CDDs conducted pre-MDA census and updated the community register to reflect those that had left the community, those that had joined the community and the newly born. MDA details were captured in the registers. After each MDA, the details were summarised on village reporting forms by CDDs and submitted to the supervising district health workers, who in turn summarised and submitted the reporting forms to include all villages they covered to DHMTs. Each DHMT then submitted the district summary report to the NTDP that collated results from all districts.

\section{Baseline surveys and sentinel sites}

Baseline data were collected during 1988-2004 from 177 sites as illustrated in Fig. 1. The sites were randomly selected from all 14 districts of Sierra Leone based on their closeness to the ten major rivers and their tributaries [20]. To monitor the impact on the onchocerciasis prevalence and MF density after five rounds of MDA, 46 sites with high prevalence (MF prevalence $240 \%$ ) from the 177 baseline sites were selected as sentinel sites. Seven of the 46 villages could not be located through field visits and had to be dropped from the list. The disappearance of villages was not unusual in the post-war context as much displacement and migration had occurred during the war. Accordingly, 39 sites (38 sites with MF prevalence $\geq 40 \%$ and one site below $40 \%$ ) in the hyper- and meso-endemic areas were finally selected as the sentinel sites and a cross-sectional survey was conducted in 2010-11. 


\section{Study population and parasitological detection}

$O$. volvulus infection was determined in convenience samples using the method of microscopic examination of skin snips for the presence of $O$. volvulus microfilariae according to $\mathrm{WHO} / \mathrm{APOC}$ recommendations. Skin snips were done in all selected villages 11-12 months after the last MDA. All volunteers/participants aged one year or above (at baseline) or five years and above (2010 survey) in each site were eligible for inclusion in the study without discrimination on gender, social status, religion or ethnicity. They were asked for identification data, including name, age, sex, occupation, and number of years they were resident in the village. Two skin biopsies were obtained from the right and left iliac crests of each participant. A $2 \mathrm{~mm}$ Holth corneoscleral punch was used to obtain the two bloodless skin snip biopsies. The scleral punch was sterilised with sodium hypo-chlorite solution and distilled water and then autoclaved for 15 min after taking biopsies from each participant.

The samples were microscopically examined for the presence of $O$. volvulus microfilariae after incubation for 30 min in distilled water. Negative skin snip samples were then kept in saline solution for another $24 \mathrm{~h}$ and microscopically re-examined. The number of microfilariae was counted and expressed as the number of microfilariae per snip, and the results were recorded for each person examined. For quality control, experts recruited by OCP and later APOC that worked in the field with the NOCP survey teams examined all positive slides and $10 \%$ of the negative slides.

\section{Statistical analysis}

Results were entered into MS Excel and analysed in SPSS (IBM, Version 19, USA). Prevalence and density of MF were calculated by district, sex and age group, and were compared with the baseline data from the same 39 sites. The $95 \%$ confidence intervals (CIs) for prevalence were calculated using the Wilson score method without continuity correction [23]. The arithmetic mean MF density of infection with $95 \% C I$ was calculated for the total population examined (both positives and negatives) and for the positives only. The Chi-squared test was used to compare the differences in prevalence, and the Kruskal-Wallis test was used to compare the differences in MF density. Treatment coverage was calculated according to the WHO guidelines and reported using two indicators: epidemiological coverage and programme coverage [24]. The total population used was the total number of people registered during the pre-MDA census CDDs conducted each year. The distribution map of the unadjusted point prevalence of the 39 villages used as sentinel sites were produced using ArcGIS software version 10.4 (ESRI, Redlands, US) [25, 26].

\section{Results}

MDA results 2005-2009

In 2005-2007, ivermectin was distributed annually to a total of 8451 villages that were meso- and hyperendemic for onchocerciasis, though about $50 \%$ of these villages conducted integrated onchocerciasis/LF MDA with ivermectin and albendazole in 2007. Between 2008 and 2009 ivermectin together with albendazole was distributed annually in the 12 provincial districts for treatment of both LF and onchocerciasis. The treatment area included the 8451 meso- and hyper-endemic onchocerciasis villages that received prior CDTI as well as the hypo-endemic villages that had not been treated under CDTI. Geographic coverage for onchocerciasis treatment of meso- and hyper-endemic communities in these districts during this period was $100 \%$. As described previously, treatment coverage in 2003 and 2004 was ineffective and is not shown here. Table 2 shows treatment coverage between 2005 and 2009. Except for 2005, treatment coverage in other years for all districts was above the minimum required threshold.

\section{Onchocerciasis situation at baseline \\ MF prevalence}

The results of surveys at baseline are shown in Table 3 . At baseline, a total of 7116 people were tested in all the 39 villages: $3461(48.6 \%)$ males and 3655 (51.4\%) females. Geographical locations of the 39 sentinel sites are shown in Fig. 2. The average baseline MF prevalence from the sentinel sites within each district varied from $39.01 \%$ (95\% CI: $36.17-41.91 \%$ ) to $61.94 \%$ (95\% CI: 54 . 09-69.20\%). MF prevalence in the northern districts (Kambia, Tonkolili, Koinadugu, Bombali and Port Loko) tended to be higher than prevalence in the southern districts (Moyamba, Bo, Pujehun and Bonthe), while the prevalence in the eastern districts was relatively the lowest (Kailahun, Kenema and Kono). The overall MF prevalence among all 7116 participants was 53.09\% (95\% CI: $51.93-54.25 \%)$. The MF prevalence was higher in males $(55.19 \%, 95 \%$ CI: $53.52-56.84 \%)$ than in females (51.11\%, 95\% CI: 49.49-52.73\%) $(P<0.001)$. Among different age groups, the MF prevalence was lowest in 19 years (11.05\%, 95\% CI: 9.80-12.43\%), increased sharply to $50.57 \%$ (95\% CI: $47.87-53.26 \%$ ) in $10-19$ years, peaked at $82.31 \%$ (95\% CI: $79.39-84.90 \%)$ in $40-49$ years, and then dropped to $76.17 \%$ (95\% CI: $72.84-79.20 \%$ ) in 60 years and aobve.

\section{MF density}

Among all 7116 people tested, the overall arithmetic mean density (AMD-all) of MF was 15.33 microfilariae (mf)/snip (95\% CI: 14.43-16.24 mf/snip), $21.11 \mathrm{mf} / \mathrm{snip}$ (95\% CI: 19.49-22.72 mf/snip) in males and $9.94 \mathrm{mf} / \mathrm{snip}$ (95\% CI: $9.09-10.79 \mathrm{mf} / \mathrm{snip})$ in females $(P<0.001)$. 


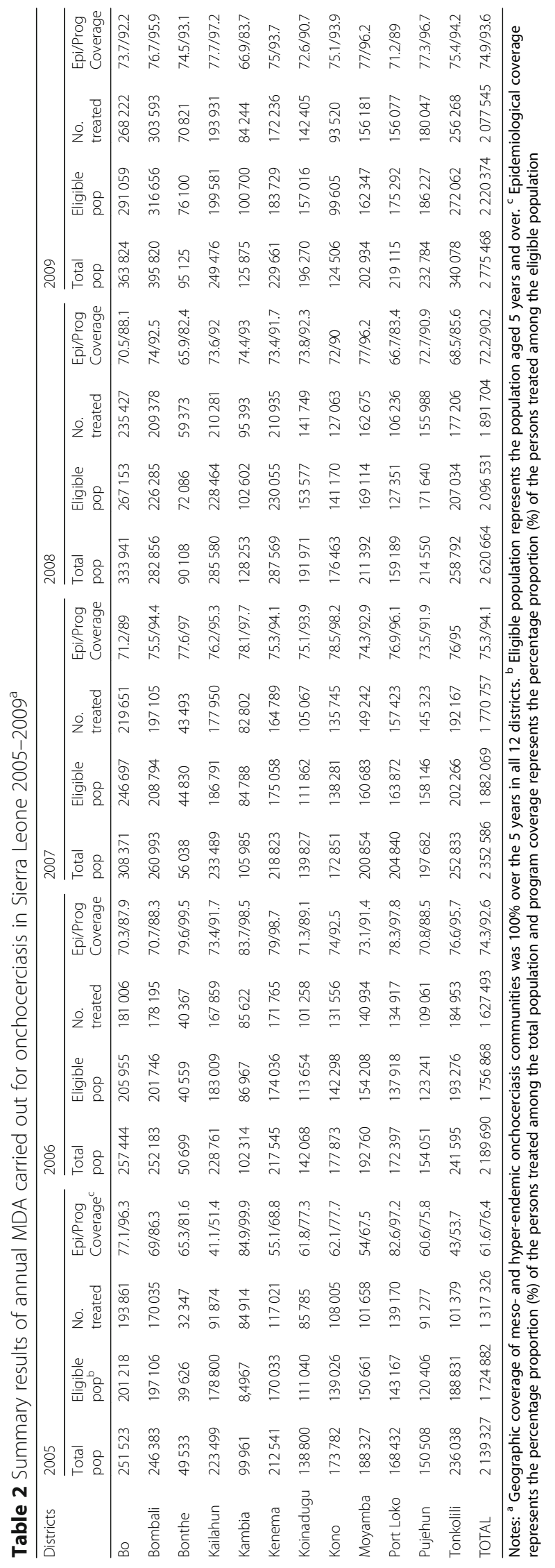




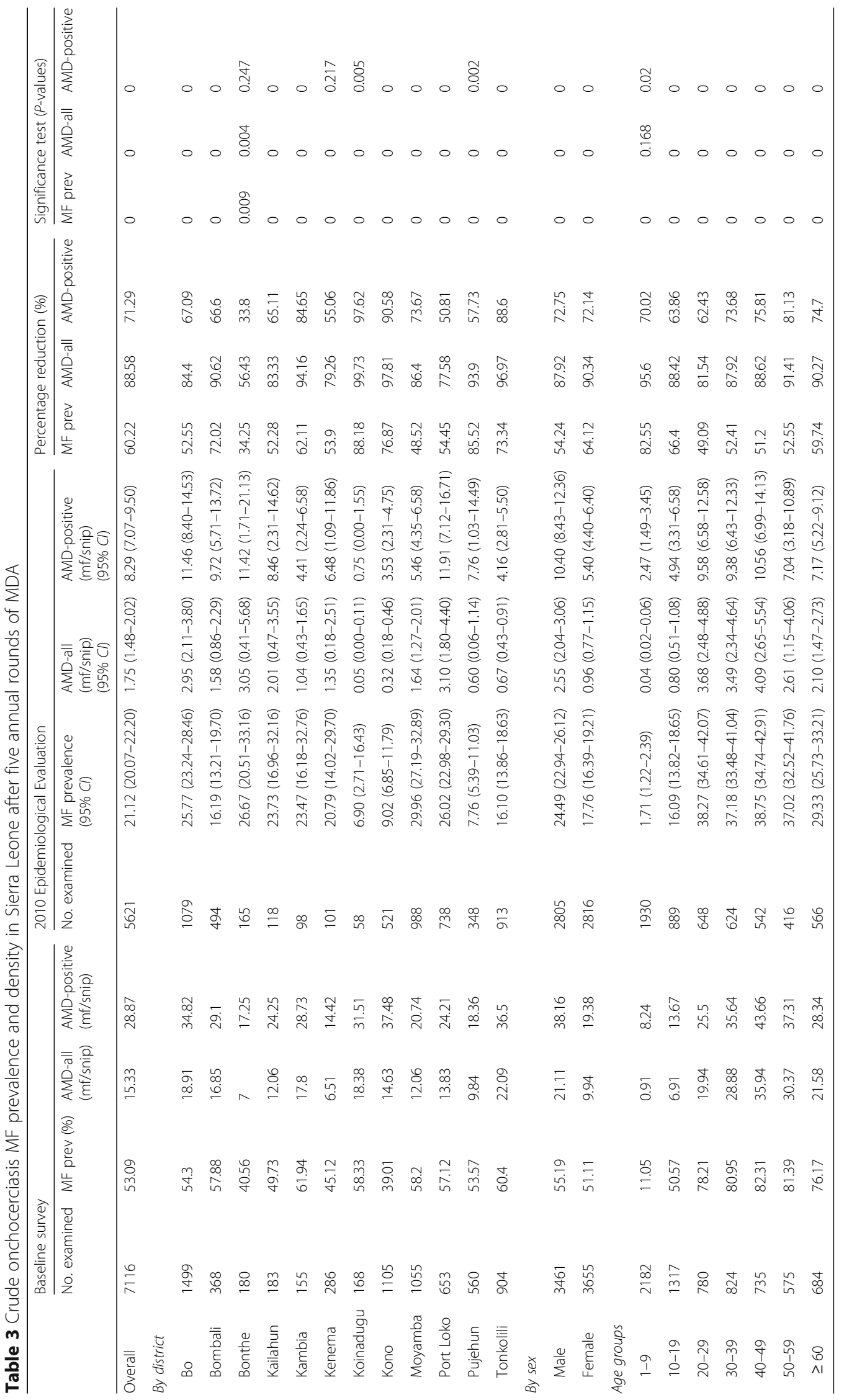




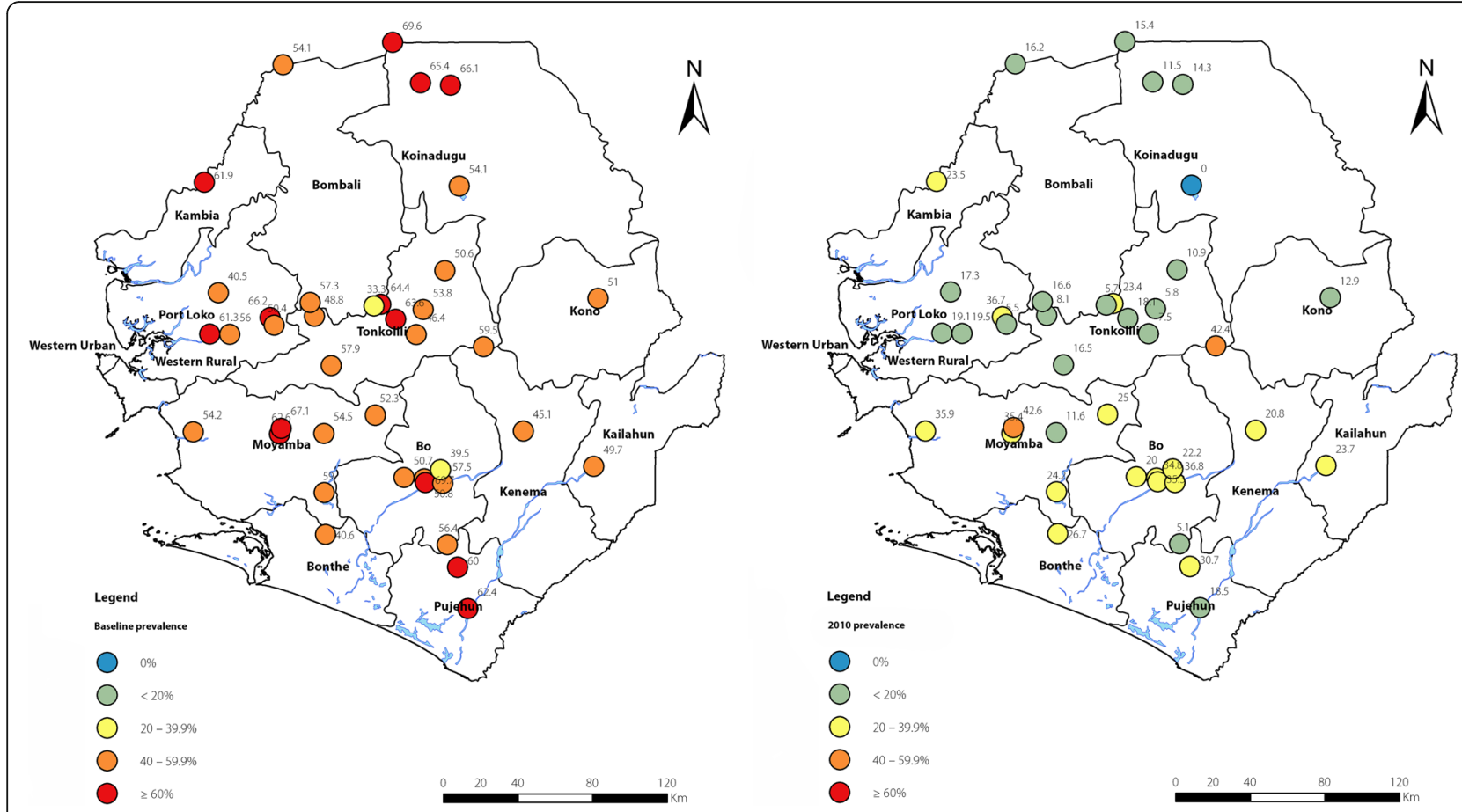

Fig. 2 Unadjusted point prevalence of onchocerciasis at the 39 sentinel sites at baseline (1988-2004, on the left) and in 2010 (on the right). Numbers represent the actual precentage point MF prevalence at each sentinel site

AMD-all ranged from $6.51 \mathrm{mf} / \mathrm{snip}$ (95\% CI: 3.47-9.54 $\mathrm{mf} / \mathrm{snip}$ ) in Kenema district to $22.09 \mathrm{mf} / \mathrm{snip}$ (95\% CI: 19.28-24.91 mf/snip) in Tonkolili district. Among the 3778 people that tested MF positive, the arithmetic mean density (AMD-positive) was $28.87 \mathrm{mf} / \mathrm{snip}$ (95\% CI: $27.29-30.46 \mathrm{mf} / \mathrm{snip}$ ), $38.16 \mathrm{mf} / \mathrm{snip}$ (95\% CI: $35.47-$ $40.84 \mathrm{mf} / \mathrm{snip}$ ) in males and $19.38 \mathrm{mf} / \mathrm{snip}$ (95\% CI: 17.85-20.92 $\mathrm{mf} / \mathrm{snip})$ in females $(P<0.001)$. The AMDpositive ranged from $14.42 \mathrm{mf} / \mathrm{snip}$ (95\% CI: 7.91-20.94 $\mathrm{mf} / \mathrm{snip}$ ) in Kenema district to $37.48 \mathrm{mf} / \mathrm{snip}$ (95\% CI: $31.36-43.60 \mathrm{mf} / \mathrm{snip}$ ) in Kono district. There was no trend observed for MF prevalence between the regions for the AMD-all and AMD-positive.

The AMD-all was lowest among the age group of 19 years (0.91 mf/snip, 95\% CI: $0.51-1.30 \mathrm{mf} / \mathrm{snip})$, increased sharply to $6.91 \mathrm{mf} / \mathrm{snip}$ (95\% CI: $5.92-7.91 \mathrm{mf} /$ snip) in the age group of 10-19 years, peaked at 35.94 $\mathrm{mf} / \mathrm{snip}$ (95\% CI: $31.44-40.44 \mathrm{mf} / \mathrm{snip}$ ) in the age group of 40-49 years, and then dropped to $21.58 \mathrm{mf} / \mathrm{snip}$ (95\% CI: $18.44-24.72 \mathrm{mf} / \mathrm{snip}$ ) in the age group of $\geq 60$ years. The AMD-positive followed the same pattern as AMDall (Table 3).

\section{Onchocerciasis situation in 2010 MF prevalence}

In 2010, a total of 5621 people were tested in all the 39 villages: 2805 (49.9\%) males and 2816 (50.1\%) females. The overall MF prevalence was $21.12 \%$ (95\% CI: $20.07-$
22.20\%): $24.49 \%$ (95\% CI: 22.94-26.12\%) in males and 17.76\% (95\% CI: $16.39-19.21 \%$ ) in females. Point MF prevalence at each sentinel site showed a general trend of decline in prevalence across the country from the baseline, which was more evident in the northern part of the country (Fig. 2). Compared with the baseline, overall MF prevalence dropped by $60.22 \%(P<0.001)$ : $54.24 \%$ among males $(P<0.001)$ and $64.12 \%$ among females $(P<0.001)$. The MF prevalence among districts ranged from $6.90 \%$ (95\% CI: 2.71-16.43\%) in Koinadugu district to $29.96 \%$ (95\% CI: $27.19-32.89 \%$ ) in Moyamba district. Reduction in MF prevalence among districts was, in general, greater than 50\% $(P<0.05)$, except for Bonthe and Moyamba districts which had $34.25 \%(P<0.01)$ and $48.52 \%$ $(P<0.001)$ reductions in prevalence respectively. Reductions in MF prevalence in Koinadugu and Pujehun districts were both over $80 \%(P<0.001)$. For the age groups, the MF prevalence showed similar age pattern as at the baseline but was at a significantly reduced level (Table 3). Among the age groups, the highest reduction in MF prevalence (82.55\%) was recorded in 1-9 years followed by the reduction in $10-19$ years $(66.40 \%)$.

\section{MF density}

The overall AMD-all was $1.75 \mathrm{mf} / \mathrm{snip}$ (95\% CI: 1.48-2.02 $\mathrm{mf} / \mathrm{snip}$ ) and the AMD-positive was $8.29 \mathrm{mf} / \mathrm{snip}$ (95\% CI: $7.07-9.50 \mathrm{mf} / \mathrm{snip})$, a significant drop of $88.58 \%$ $(P<0.001)$ and $71.29 \%(P<0.001)$ from the baseline, 
respectively. Compared to the baseline, the AMD-all was $2.55 \mathrm{mf} / \mathrm{snip}$ (95\% CI: $2.04-3.06 \mathrm{mf} / \mathrm{snip}$ ) in males, a decrease of $87.92 \%(P<0.001)$, and $0.96 \mathrm{mf} / \mathrm{snip}$ (95\% CI: $0.77-1.15 \mathrm{mf} / \mathrm{snip})$ in females, a decrease of $90.34 \%$ $(P<0.001)$. The AMD-positive was $10.40 \mathrm{mf} / \mathrm{snip}(95 \%$ CI: $8.43-12.36 \mathrm{mf} / \mathrm{snip})$ in males, a decrease of $72.75 \%$ $(P<0.001)$ and $5.40 \mathrm{mf} / \mathrm{snip}(95 \% C I: 4.40-6.40 \mathrm{mf} /$ snip) in females, a decrease of $72.14 \%(P<0.001)$. There was a significant difference in AMD between males and females $(P<0.05)$. Among districts, the AMD-all ranged from $0.05 \mathrm{mf} / \mathrm{snip}$ (95\% CI: 0.00-0.11 $\mathrm{mf} / \mathrm{snip}$ ) in Koinadugu district to $3.10 \mathrm{mf} / \mathrm{snip}$ (95\% CI: $1.80-4.40 \mathrm{mf} / \mathrm{snip})$ in Port Loko district, in general a significant reduction of $>70 \%(P<0.05)$ across districts, with the exception of Bonthe district, which showed a reduction of $56.43 \%(P<0.01)$. The AMD-positive ranged from $0.75 \mathrm{mf} / \mathrm{snip}$ (95\% CI: $0.00-1.55 \mathrm{mf} / \mathrm{snip}$ ) in Koinadugu district to $11.91 \mathrm{mf} / \mathrm{snip}$ (95\% CI: 7.12-16.71 $\mathrm{mf} / \mathrm{snip}$ ) in Port Loko district, in general a significant reduction of $>50 \%(P<0.05)$, with the exception of Bonthe district where the reduction was $33.80 \%(P>0.05)$. Among the age groups, the AMD-all and AMD-positive showed similar age distribution pattern as the baseline, however, there was in general $>80 \%$ reduction for AMD-all and $>60 \%$ reduction for AMD-positive (Table 3).

\section{Discussion}

The following distinct epidemiological patterns were determined in the MF prevalence from the survey data: 1 ) higher in males than in females, 2) lowest in 1-9 years followed by that in 10-19 years, and 3) higher in the northern districts than in the southern and eastern districts. Distinct epidemiological patterns were also noted in the MF density. AMD-all or AMD-positive was higher in males than in females, was lowest among the age group of 1-9 years followed by that in the age group of 10-19 years, and was highest among the age group of $40-49$ years.

In general, males had higher $O$. volvulus infection levels than females. Although MF prevalence in males was only slightly higher than in females, the MF density for all surveyed and positive-only participants was about twice as high in males as in females. The similar male/ female differences in onchocerciasis prevalence, density of infection and clinical disease have been discussed by many authors [27-33]. However, it should be noted that Gbakima and Sahr detected no difference in O. volvulus infection between males and females in their Sierra Leone study [34]. It has been suggested that higher $O$. volvulus infection in males, compared to females, may be due to increased exposure to black flies among males through fishing and other activities that take place in close proximity to rivers $[29,35,36]$.
Lower age groups (1-9 years and 10-19 years) had the lowest MF prevalence and density even within highly endemic areas. MF prevalence and density were highest in the age group of 40-49 years. This age trend was similar to the results of a study in Nigeria that found O. volvulus MF prevalence and intensity increasing with age [28]. This may be explained by the fact that adults with growing age have more exposure to the bite of infected black flies. It is reported that maternal O. volvulus infection can be transmitted, even in utero, to their children and that children of onchocerciasis-infected mothers are more likely to be infected with onchocerciasis [37, 38]. The fact that females in our surveyed villages were less infected than men suggests that children in these villages may have had less exposure to the bite of infected black flies or in utero transmission.

It has been suggested that the regional and district variations of onchocerciasis prevalence at baseline could be linked with vector ecology and density, which also depend on the distance of villages studied from the river basin and the geography of the river [39]. Previous studies in Sierra Leone showed that O. volvulus MF prevalence was higher in the forest (71.8\%) than in Savannah villages $(51.9 \%)$ [30]. In general, the eastern region has the forest strain of the O. volvulus parasite, characterised by low intensity of infection, mild skin disease and relatively low blindness rate; the southern region has a mixture of forest and Savannah strains of the parasite, characterised by high infection intensity, mild skin disease and relatively higher blindness rate (sometimes higher than blindness rates recorded in the Savannah area); and the northern region has the Savannah strain of the parasite, characterised by high infection intensity, mild skin disease, and relatively high blindness rate [14, 34]. Our survey data may have reflected these.

The 2010 evaluation revealed a significant decrease in onchocerciasis MF prevalence and density in the same 39 sentinel villages after just five rounds of annual MDA. The same epidemiological dynamics as the baseline was observed in the 2010 evaluations, i.e. MF prevalence was higher in males than in females and MF density was twice as high in males as in females. The regional disease distribution observed at baseline (the northern districts with relatively higher prevalence, followed by the southern and then the eastern having the lowest relative prevalence) appeared to have changed significantly after five years of MDA. In 2010, the northern districts had the lowest average MF prevalence relative to the eastern and southern districts. This observation could not be explained by differences in treatment coverage (similar in all the regions) but may be due to the responsiveness of different parasite strains to treatment and further studies are needed to clarify this. The significant reduction in $O$. volvulus infection after five years of MDA is similar to the 
results from other countries. In Cameroon, onchocerciasis MF prevalence in the Fundong health district decreased from $60.0 \%$ to $3.5 \%$ after six rounds of continuous MDA using the CDTI strategy [40]. After seven years (19952001) of ivermectin treatment in three endemic villages of the Etung Local Government Area of Lower Cross River Basin, Nigeria, MF prevalence decreased from $63.3 \%$ at baseline to $39.3 \%$ and community MF density dropped from 7.11 to $2.31 \mathrm{mf} / \mathrm{snip}$ [38].

Significant progress has been made on onchocerciasis elimination in Sierra Leone. Effective epidemiological and programme coverage in all endemic areas was reached and has been maintained since 2005. By 2017, considering the missed MDA due to Ebola virus disease in 2014, a total of 10 rounds of effective MDA have been completed. Per the current WHO recommendations, 12-15 years of MDA are needed for onchocerciasis elimination [22]. It is anticipated that additional 2-5 annual rounds of MDA may bring Sierra Leone to the point of stopping MDA for onchocerciasis. However, there are currently no guidelines on the prevalence threshold and methodology for deciding when to move to stop-MDA evaluation. While continuing with the ivermectin MDA in the 12 districts, the NTDP has established a national onchocerciasis elimination committee (NOEC), per WHO recommendations, which will review the programme progress and recommend a way forward for the NTDP on onchocerciasis elimination.

There are certain limitations in this study. First, the baseline data were obtained over a long period. Some villages were studied pre-war in 1988-1990 and others were studied post-war in 2002-2004 with a gap of about 11 years. It is possible that the epidemiological situation may have changed in villages studied in the earlier years. This is exemplified by the fact that 7 of the originally selected villages could not be traced. Therefore, comparing with the baseline data collected pre-war, the reduction in infection seen in 2010 may have not been entirely due to the impact of MDA. However, we tried to minimize this effect by including more villages with more recent data (31 sites with data from 2002 to 2004 and eight sites with data from 1998 to 1990). Villages with older data (1988-1990) were only selected when more recent data (2002-04) were not available for a district. Second, in the 2010 evaluation, only persons aged five years and above were studied while all persons aged over one year old were studied at baseline. This resulted in fewer children aged 1-9 years examined in the 2010 evaluation. The decision not to study children below five years of age was based on the high refusal rate to skin snip in this age group observed in communities during the baseline studies. Newly developed serological tools (OV16 rapid diagnostic tests [RDTs] and OV16 ELISA) should be considered for better compliance in future evaluations [41-43]. Third, the sentinel sites surveyed in 2010 did not include hypoendemic villages. As the focus for onchocerciasis has shifted from control to elimination and hypo-endemic villages have been treated through LF MDA. It is essential to include hypo-endemic villages in future evaluations to have a full picture of the current onchocerciasis for national programme decision-making.

\section{Conclusions}

There was a significant reduction of onchocerciasis MF prevalence and MF density across the 12 rural onchocerciasis-endemic districts of Sierra Leone after five annual MDAs. The results suggested that the onchocerciasis elimination programme in Sierra Leone was on course to reach the objective of eliminating onchocerciasis in Sierra Leone by the year 2025. However, MDA needs to continue in all 12 districts with required treatment coverage to reach the goal of interrupting transmission. Further evaluations across the country are needed to assist the NTDP with programme decision-making.

\section{Additional file}

Additional file 1: Multilingual abstracts in the five official working languages of the United Nations. (PDF 483 kb)

\section{Abbreviations}

AMD-all: Arithmetic mean density for all persons tested; AMDpositive: Arithmetic mean density among people that tested MF positive; APOC: African Programme for Onchocerciasis Control; CDD: Communitydirected drug distributor; CDTI: Community-Directed Treatment with Ivermectin; Cl: Confidence interval; DHMT: District Health Management Team; LF: Lymphatic filariasis; MDA: Mass drug administration; mf: Microfilariae; MF: Microfilaridermia; MOHS: Ministry of Health and Sanitation; NOCP: National Onchocerciasis Control Program; NOEC: National Onchocerciasis Elimination Committee; NTD: Neglected tropical diseases; NTDP: Neglected Tropical Disease Program; OCP: Onchocerciasis Control Programme in West Africa; RDT: Rapid diagnostic test; SIZ: Special Intervention Zones; USAID: United States Agency for International Development; WHO: World Health Organisation

\section{Acknowledgements}

The NOCP/NTDP in Sierra Leone received financial and technical support from the $\mathrm{WHO} / \mathrm{OCP}$ and $\mathrm{WHO} / \mathrm{APOC}$ until their respective closure. The NTDP has since 2008 been supported by the United States Agency for International Development (USAID) through a grant to Helen Keller International under Cooperative Agreement No. GHS-A-00-06-00006-00 with the End in Africa project managed by FHI360. Sightsavers has also provided financial support to the onchocerciasis control. The Centre for Neglected Tropical Diseases, Liverpool supported the national NTD laboratory. Special thanks go to the technical team leaders: A. Tia and S. Saffa.

\section{Funding}

All the studies reported in this paper were funded by WHO through OCP or APOC, who also provided technical support in the design, implementation (quality control) and interpretation of the baseline data.

\section{Availability of data and materials}

All data generated or analysed during this study are included in this published article. The dataset analysed is available from the corresponding author on reasonable request and can be made available with permission from the MOHS Sierra Leone. 


\section{Authors' contributions}

JBK (2005-2009) and SS (2009-2014) are former programme managers of the NOCP/NTDP Sierra Leone, designed and coordinated the studies and initial reports. AC, JP, MB, MS participated in the field work. JBK and $Y Z$ wrote the manuscript. BK, MHH, MJB revised the paper. JBK and YZ conducted the initial analysis. YZ conducted the final data analysis and spatial analysis. All authors reviewed and approved the final manuscript.

\section{Ethics approval and consent to participate}

The surveys were part of the monitoring and evaluation activities of the NOCP of the MOHS, Sierra Leone. Ethical approval for data collection was obtained from the Ethics Committee of the MOHS, Sierra Leone. Upon arrival at the selected communities, the investigating teams met with community leaders and explained the nature of their work and the objectives of the study. Volunteers participated in the studies after informed consent was verbally obtained and recorded by the team leader as literacy rates are low in Sierra Leone. Consent was obtained from participants themselves, or from the parents of all participants below the age of 15 years. Data collection was conducted such that participants will remain anonymous during data entry and analysis. No individual's identity can be revealed upon publication.

\section{Consent for publication}

Not applicable.

\section{Competing interests}

The authors declare that they have no competing interests.

\section{Author details}

'Family Health International (FHI) 360, Ghana Country Office, Accra, Ghana. ${ }^{2}$ National Neglected Tropical Disease Control Programme, Ministry of Health and Sanitation, Freetown, Sierra Leone. ${ }^{3}$ Centre for Neglected Tropical Diseases, Liverpool School of Tropical Medicine, Liverpool, UK. ${ }^{4}$ Helen Keller International, Freetown, Sierra Leone. ${ }^{5}$ Helen Keller International, Regional Office for Africa, Dakar, Senegal. ${ }^{6}$ European \& Developing Countries Clinical Trials Partnership (EDCTP), Medical Research Council, Cape Town, South Africa.

\section{Received: 9 August 2017 Accepted: 20 March 2018}

\section{Published online: 06 April 2018}

\section{References}

1. Basanez MG, Pion SD, Churcher TS, Breitling LP, Little MP, Boussinesq M. River blindness: a success story under threat. PLoS Med. 2006;3:e371.

2. Gloeckner C, Garner AL, Mersha F, Oksov Y, Tricoche N, Eubanks LM, et al. Repositioning of an existing drug for the neglected tropical disease onchocerciasis. Proc Natl Acad Sci U S A. 2010;107:3424-9.

3. Traore MO, Sarr MD, Badji A, Bissan Y, Diawara L, Doumbia K, et al. Proof-ofprinciple of onchocerciasis elimination with ivermectin treatment in endemic foci in Africa: final results of a study in Mali and Senegal. PLoS Negl Trop Dis. 2012;6:e1825.

4. Conceptual and operational framework of onchocerciasis elimination with ivermectin treatment. World Health Organization. 2010. http://www.who.int/ apoc/oncho_elimination_report_english.pdf. Accessed 4 Jan 2018.

5. Crump A, Morel $\mathrm{CM}$, Omura S. The onchocerciasis chronicle: from the beginning to the end? Trends Parasitol. 2012;28:280-8.

6. Taylor HR. The power of one- stemming the tide of river blindness: the early years of ivermectin. Med J Aust. 2003;179:617-9.

7. Third Progress Report on the London Declaration. Uniting to Combat Neglected Tropical Diseases 2015. http://unitingtocombatntds.org/resource/ 2015-third-progress-report-london-declaration. Accessed 4 Jan 2018.

8. World Health Organization. Progress towards eliminating onchocerciasis in the WHO region of the Americas: verification of elimination of transmission in Guatemala. Progress report on the elimination of human onchocerciasis, 2015-2016. Wkly Epidemiol Rec. 2016;43:501-16.

9. Molyneux DH, Hopkins A, Bradley MH, Kelly-Hope LA. Multidimensional complexities of filariasis control in an era of large-scale mass drug administration programmes: a can of worms. Parasit Vectors. 2014;7:363.

10. Diawara L, Traore MO, Badji A, Bissan Y, Doumbia K, Goita SF, et al. Feasibility of onchocerciasis elimination with ivermectin treatment in endemic foci in Africa: first evidence from studies in Mali and Senegal. PLoS Negl Trop Dis. 2009;3:e497.
11. Hodgkin C, Molyneux DH, Abiose A, Philippon B, Reich MR, Remme JH, et al. The future of onchocerciasis control in Africa. PLoS Negl Trop Dis. 2007;1:074.

12. Tekle AH, Elhassan E, Isiyaku S, Amazigo UV, Bush S, Noma M, et al. Impact of long-term treatment of onchocerciasis with ivermectin in Kaduna state, Nigeria: first evidence of the potential for elimination in the operational area of the African Programme for onchocerciasis control. Parasit Vectors. 2012;5:28

13. Bockarie MJ, Kelly-Hope LA, Rebollo M, Molyneux DH. Preventive chemotherapy as a strategy for elimination of neglected tropical parasitic diseases: endgame challenges. Philos Trans R Soc Lond Ser B Biol Sci. 2013; 368:20120144.

14. Blacklock DB. The development of Onchocerca volvulus in Simulium damnosum. Ann Trop Med Parasitol. 1926:29:1-47.

15. Dadzie KY, De Sole G, Remme J. Ocular onchocerciasis and the intensity of infection in the community. IV. The degraded forest of Sierra Leone. Trop Med Parasitol. 1992;43:75-9.

16. The Onchocerciasis Control Programme in West Africa : an example of effective public health management. World Health Organization. 1994. http://apps.who. int/iris/bitstream/10665/39261/1/WHO_PHA_1.pdf. Accessed 4 Jan 2018.

17. Yameogo L. Special intervention zones. Ann Trop Med Parasitol. 2008; 102(Suppl 1):23-4.

18. Hodges ME, Koroma JB, Sonnie M, Kennedy N, Cotter E, MacArthur C. Neglected tropical disease control in post-war Sierra Leone using the onchocerciasis control Programme as a platform. Int Health. 2011;3:69-74.

19. Fobi G, Yameogo L, Noma M, Aholou Y, Koroma JB, Zoure HM, et al. Managing the fight against onchocerciasis in Africa: APOC experience. PLoS Negl Trop Dis. 2015;9:e0003542.

20. Ministry of Health and Sanitation. Annual Report of the National Onchocerciasis Control Programme. Freetown. Sierra Leone: Ministry of Health and Sanitation; 2005. p. 2006.

21. Curriculum and training module on the community-directed intervention (CDI) strategy for faculties of medicine and health sciences (2nd edition). African Programme for Onchocerciasis Control, World Health Organization. 2012. http://www.who.int/apoc/publications/EN_CDI_Manual_final.pdf?ua= 1. Accessed 4 Jan 2018.

22. Guidelines for stopping mass drug administration and verifying elimination of human onchocerciasis: Criteria and procedures. World Health Organization. 2016. http://www.who.int/onchocerciasis/resources/ 9789241510011/en/. Accessed 4 Jan 2018.

23. Newcombe RG. Two-sided confidence intervals for the single proportion: comparison of seven methods. Stat Med. 1998;17:857-72.

24. World Health Organization. Monitoring and epidemiological assessment of mass drug administration: a manual for national elimination programmes. Geneva: World Health Organization; 2011.

25. Zoure HG, Wanji S, Noma M, Amazigo UV, Diggle PJ, Tekle AH, et al. The geographic distribution of Loa loa in Africa: results of large-scale implementation of the rapid assessment procedure for Loiasis (RAPLOA). PLoS Negl Trop Dis. 2011;5:e1210.

26. Koroma JB, Bangura MM, Hodges MH, Bah MS, Zhang Y, Bockarie MJ. Lymphatic filariasis mapping by immunochromatographic test cards and baseline microfilaria survey prior to mass drug administration in Sierra Leone. Parasit Vectors. 2012;5:10.

27. Wyatt GB. Onchocerciasis in Ibarapa, western state, Nigeria. Ann Trop Med Parasitol. 1971;65:513-23.

28. Opara KN, Fagbemi BO. Population dynamics of onchocerca volvulus microfilariae in human host after six years of drug control. J Vector Borne Dis. 2008:45:29-37.

29. Courtright $P$, Johnston $K$, Chitsulo L. A new focus of onchocerciasis in Mwanza District, Malawi. Trans R Soc Trop Med Hyg. 1995;89:34-6.

30. McMahon JE, Sowa SI, Maude GH, Kirkwood BR. Onchocerciasis in Sierra Leone.2: a comparison of forest and savanna villages. Trans R Soc Trop Med Hyg. 1988;82:595-600

31. MCMahon JE, Sowa SC, Maude GH, Hudson CM, Kirkwood BR. Epidemiological studies of onchocerciasis in forest villages of Sierra Leone. Trop Med Parasitol. 1988;39:251-9.

32. McMahon JE, Sowa SI, Maude GH, Hudson CM, Kirkwood BR. Epidemiological studies of onchocerciasis in savanna villages of Sierra Leone. Trop Med Parasitol. 1988;39:260-8.

33. Little MP, Basanez MG, Breitling LP, Boatin BA, Alley ES. Incidence of blindness during the onchocerciasis control programme in western Africa, 1971-2002. J Infect Dis. 2004;189:1932-41. 
34. Gbakima AA, Sahr F. Filariasis in the Kaiyamba chiefdom, Moyamba District Sierra Leone: an epidemiological and clinical study. Public Health. 1996;110:169-74.

35. Brabin L. Factors affecting the differential susceptibility of males and females to onchocerciasis. Acta Leiden. 1990;59:413-26.

36. Onchocerciasis and its control : report of a WHO Expert Committee on Onchocerciasis Control [Internet]. World Health Organization. 1995. http:// apps.who.int/iris/bitstream/10665/37346/1/WHO_TRS_852.pdf. Accessed 4 Jan 2018.

37. Kirch AK, Duerr HP, Boatin B, Alley WS, Hoffmann WH, Schulz-Key H, et al. Impact of parental onchocerciasis and intensity of transmission on development and persistence of Onchocerca volvulus infection in offspring: an 18 year follow-up study. Parasitology. 2003;127:327-35.

38. Brinkmann UK, Kramer P, Presthus GT, Sawadogo B. Transmission in utero of microfilariae of Onchocerca volvulus. Bull World Health Organ. 1976;54:708-9.

39. Brieger WR, Ososanya OO, Kale OO, Oshiname FO, Oke GA. Gender and ethnic differences in onchocercal skin disease in Oyo state, Nigeria. Tropical Med Int Health. 1997;2:529-34.

40. Kamga HL, Shey DN, Assob JC, Njunda AL, Nde Fon P, Njem PK. Prevalence of onchocerciasis in the Fundong Health District, Cameroon after 6 years of continuous community-directed treatment with ivermectin. Pan Afr Med J. 2011;10:34.

41. Weil GJ, Steel C, Liftis F, Li BW, Mearns G, Lobos E, et al. A rapid-format antibody card test for diagnosis of onchocerciasis. J Infect Dis. 2000; 182:1796-9.

42. Lipner EM, Dembele N, Souleymane S, Alley WS, Prevots DR, Toe L, et al. Field applicability of a rapid-format anti-Ov-16 antibody test for the assessment of onchocerciasis control measures in regions of endemicity. J Infect Dis. 2006;194:216-21.

43. Golden A, Stevens EJ, Yokobe L, Faulx D, Kalnoky M, Peck R, et al. A recombinant positive control for serology diagnostic tests supporting elimination of Onchocerca volvulus. PLoS Negl Trop Dis. 2016:10:e0004292.

\section{Submit your next manuscript to BioMed Central and we will help you at every step:}

- We accept pre-submission inquiries

- Our selector tool helps you to find the most relevant journal

- We provide round the clock customer support

- Convenient online submission

- Thorough peer review

- Inclusion in PubMed and all major indexing services

- Maximum visibility for your research

Submit your manuscript at www.biomedcentral.com/submit 